\title{
Transient Response Characteristics of Separated Flow and Heat Transfer in Enlarged Rectangular Channel
}

\author{
Hiroyuki Yoshikawa $^{1 *}$, Shota Shirakura ${ }^{2}$, Mizue Munekata ${ }^{1}$ \\ ${ }^{1}$ Department of Mechanical System Engineering, Kumamoto University, Kumamoto, Japan \\ ${ }^{2}$ Graduate School of Science and Technology, Kumamoto University, Kumamoto, Japan \\ Email: ${ }^{*}$ yoshi@kumamoto-u.ac.jp
}

Received September 18, 2012; revised October 30, 2012; accepted November 9, 2012

\begin{abstract}
Numerical results of three-dimensional separated flow and heat transfer in an enlarged rectangular channel are presented in this paper. The expansion ratio and aspect ratio of the channel are 2.0 and 8.0, respectively. Reynolds number of the flow is 200 and it is over the critical Reynolds number. Over the value, the flow in the symmetric channel becomes to deflect to one side of the walls. Transient response characteristics of the flow and heat transfer in the channel with the fully developed flow imposed one cycle of a pulsating fluctuation at the inlet are investigated. Vortex structure generated in the channel is visualized with a helicity isosurface. In the case of the fluctuation of Strouhal number 0.05 , small streamwise vortices appear near the side walls and slightly upstream of the reattachment region of the short separation bubble. The vortices elongate and shed some vortices. These vortices attract some pairs of the streamwise vortices near the reattachment region quickly and they drift downstream along the side walls. They are inclined from the walls and are decaying gradually. It is clarified that high Nusselt number area appears and shifts downstream in accordance with the root of the vortices.
\end{abstract}

Keywords: Transient Response; Pulsating Fluctuation; Symmetric Enlarged Channel; Asymmetric Separated Flow; Heat Transfer

\section{Introduction}

Recently, prediction of separated flow and heat transfer in an enlarged channel is very important in relation to many types of fluid machinery and heat exchangers, and there have been so many experimental and numerical investigations [1-14]. It has been clarified in these studies that the flow in a two-dimensional symmetrically enlarged channel becomes asymmetric over the critical Reynolds number. The cause of becoming a steady asymmetric flow can be explained by the Coanda effect. The pressure decreases owing to an increase of the velocity near the lower or upper wall in the channel. The flow is biased to the lower pressure side when the pressure difference is once generated across the channel, and the asymmetry of flow is stably maintained. It seems that the instability of the separated shear layer disturbs the velocity resulting in the asymmetric flow. However, the details of these phenomena have not been clarified yet.

In engineering applications, the flow channel almost usually provides the side walls, which affect greatly the flow structure resulting in the three-dimensional flow [3, 13]. Accordingly, such the flow and heat transfer may be

*Corresponding author. quite different from that obtained with the two-dimensional simulation. However, there has been little information about the three-dimensional structure of separated flow and heat transfer in an enlarged channel. Cherdron et al. [3] and Schreck and Schäfer [13] have shown the stabilizing effect of the side walls experimentally and numerically.

Several investigations regarding influences of the side walls upon the heat transfer have been performed. Yanaoka and Ota [15,16] ant Ota et al. [17] have investigated numerically a three-dimensional flow and heat transfer over a blunt flat plate in a channel and in a rectangular channel with a sudden expansion. It has been clarified that the flow in the separation bubble becomes extremely three-dimensional with an increase of the Reynolds number because of the side walls and the heat transfer is greatly enhanced near the side walls. Iwai et al. [18] have studied numerically a laminar flow and heat transfer over a backward-facing step in a rectangular duct and reported that the heat transfer is enhanced near the side walls. However, they have treated a steady asymmetric flow and there have been only a few works on an unsteady flow in the channel. The authors [19] have investigated effects of inlet flow imposed pulsating fluctuation [20] 
upon the separated flow and heat transfer in the enlarged rectangular channel. It is clarified that the pulsating fluctuation of $\mathrm{St}=0.05$ and 0.10 strongly affects on the flow in the channel and heat transfer on wall surfaces enhanced, especially on the wall covered with the long separation bubble.

However, transient flow and heat transfer with the inlet flow imposed fluctuation are not clarified in detail. The main objective of the present research is to clarify the transient response characteristics of the separated flow and heat transfer with only one cycle of a pulsating fluctuation in the enlarged rectangular channel.

\section{Fundamental Equations and Numerical Procedures}

In the present study, three-dimensional (3D) flow of an incompressible viscous fluid with constant properties is treated. Figure 1 illustrates a 3D enlarged channel along with the coordinates and several important geometrical parameters. The fully developed laminar flow enters into the inlet, but only one cycle of a pulsating fluctuation [20] is imposed initially. The flow separates at the step corner and reattaches to the downstream walls. The wall surfaces in the downstream of the step are heated. The present study simulates the forced convection and does not consider the influence of buoyancy. Fundamental equations are the continuity, momentum and energy ones.

$$
\begin{gathered}
\nabla \cdot \boldsymbol{u}=0 \\
\boldsymbol{u}_{t}+(\boldsymbol{u} \cdot \nabla) \boldsymbol{u}=-\nabla p+\frac{1}{\operatorname{Re}} \nabla^{2} \boldsymbol{u} \\
\theta_{t}+(\boldsymbol{u} \cdot \nabla) \theta=\frac{1}{\operatorname{RePr}} \nabla^{2} \theta
\end{gathered}
$$

In these equations, physical quantities are nondimen-
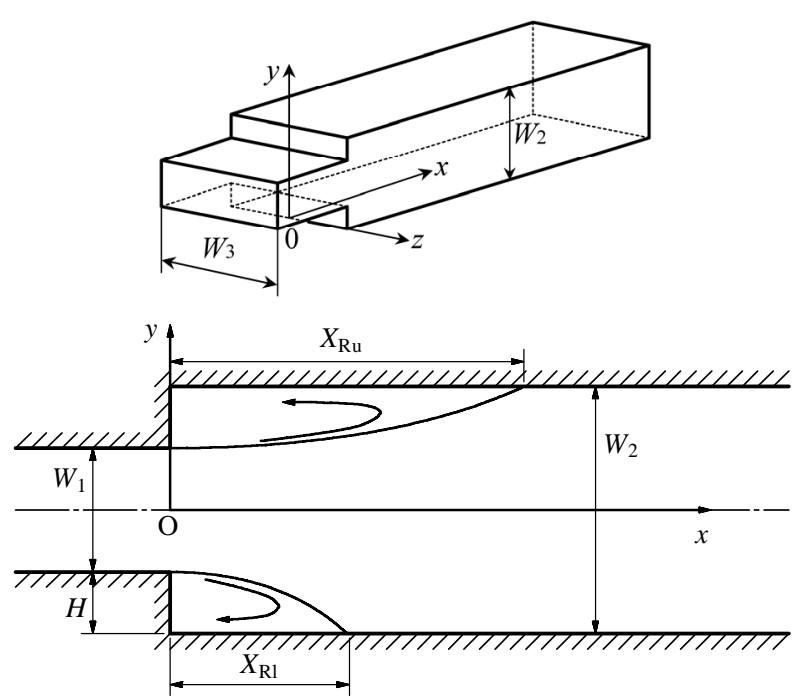

Figure 1. Flow configuration and coordinate system. sionalized by $\boldsymbol{x}^{*}=\boldsymbol{x} / H, \boldsymbol{u}^{*}=\boldsymbol{u} / U, p^{*}=p / \rho U^{2}$, $\theta^{*}=\left(\theta-\theta_{i}\right) /\left(\theta_{w_{*}}-\theta_{i}\right)$ and $t^{*}=t U / H$, respectively. The superscript ${ }^{*}$ indicates nondimensional quantities, and it is omitted in the above equations. The finite difference forms of these equations are obtained by the second-order Adams-Bashforth method for the time differentials. The fifth-order upwind difference is employed for the convection terms in order to make clear the details of flow structure, and the fourth-order central difference for the diffusion terms, and the second-order central difference for other terms. Resulting finite equations are solved using the SMAC method [21].

The numerical calculations are performed on the cases of the expansion ratio, $E R=2$, the aspect ratio, $A R=8$, the Reynolds number based on the mean flow velocity at the inlet and the step height, $\mathrm{Re}=200$, and the Prandtl number, $\operatorname{Pr}=0.71$, respectively. The computational region extends from $10 \mathrm{H}$ upstream of the step to $100 \mathrm{H}$ downstream of the step. The boundary conditions are as follows. As for the velocity, the no-slip condition is assumed on the walls and the zero gradient is used at the outlet. At the inlet, the fully developed duct flow velocity profile is presumed, though only one cycle of a pulsating fluctuation [20] is imposed initially. As for the temperature, it is assumed to be uniform at the inlet, and the lower and upper wall surfaces downstream of the step are heated under the condition of uniform temperature, and other walls are adiabatic. The second derivative is assumed to be zero at the outlet. Initial flow and temperature fields are steady state in the channel and the flow deflects downward, and after that the proper symmetric velocity profile with only one cycle of a pulsating fluctuation is imposed at the inlet.

The computational grids are generated using the Cartesian coordinates with non-uniform spacings and the grids of $251 \times 155 \times 61$ are used.

\section{Results and Discussion}

In the preliminary calculations, in order to verify the present code, numerical streamwise velocity profiles on the center plane at several streamwise cross sections are compared with the previous measured ones by Fearn et al. [7] for $E R=3, A R=24, \mathrm{Re}=186.7$, and Durst et al. [9] for $E R=2, A R=16, R e=203.3$ in Figure 2. In the case of $\operatorname{Re}=186.7$, the present results agree very well with the measured ones in the whole flow field. In both cases, the instability of shear layers separated from the step corner increases and the flow becomes asymmetric because of the pressure difference across the channel. The steady asymmetric flow is stably maintained. In the case of $\mathrm{Re}=$ 203.3, the present numerical results reasonably agree well with the measured ones, though small differences can be detected around the shear layers. It is necessary to 
confirm the grid dependency in the case of $\mathrm{Re}=203.3$ using a finer grid in order to clarify these small discrepancies. However, these comparisons show the good reliability of the present numerical scheme.

Figures 3 and 4 illustrate side and bottom views of instantaneous helicity isosurface. Two inclined vortices exist behind the step near the side walls symmetrically at $t=0$. These vortices originated by a jet like flow in steady state [18] and almost keep the shape and location. These vortices are elongated toward spanwise center of the channel on the lower wall from $t=5$. Corotating vortices appear downstream of the vortices at $t=17$. At $t=$ 20 , the initial vortices shed two vortices. Counter rotating vortices are induced in front of the downstream vortices near the side walls. The initial shed vortices slightly incline to the upper wall and upper share layer sheds two vortices at $t=25$. Downstream vortices successively induce counter rotating vortices from $t=25$ to $t=80$. The

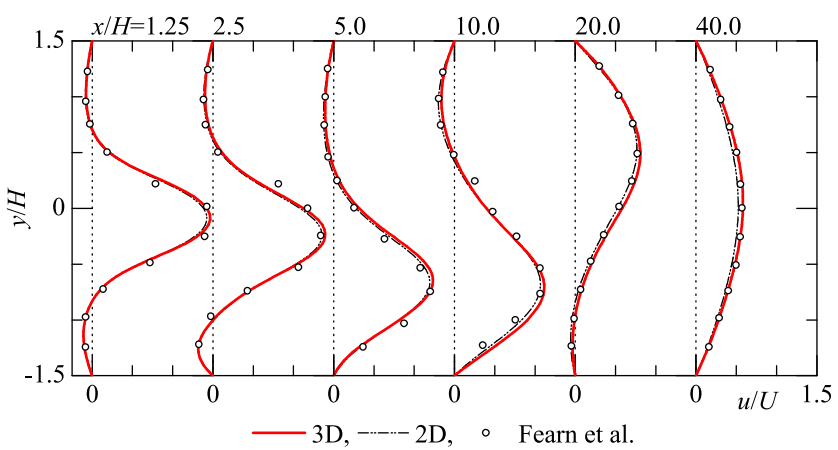

(a) vortices gradually inclined from the walls from $t=47$. The cloud of the vortices gradually decreases its intensity from $t=90$ and are divided at $x / H=60$ and $t=95$. These vortices monotonically decay and split and nearly disappear at $t=160$.

Figures 5 and $\mathbf{6}$ present instantaneous local Nusselt number distribution on lower and upper walls. The distribution within the separation bubbles is little changed. On the lower wall, heat transfer is slightly enhanced in the spanwise center region from $t=25$. Two, four and six areas of high Nusselt number exist near the side walls at $t$ = 39, 45 and 55, respectively. Inclined vortices come close to the lower wall and a pair of counter rotating vortices entrains cold fluid to the wall. These regions are identical to area of high Nusselt number. At $t=62$, high Nusselt number area appears in the spanwise center region and gradually increases the value. At $t=82$, Nusselt number takes a peak in the spanwise channel center and

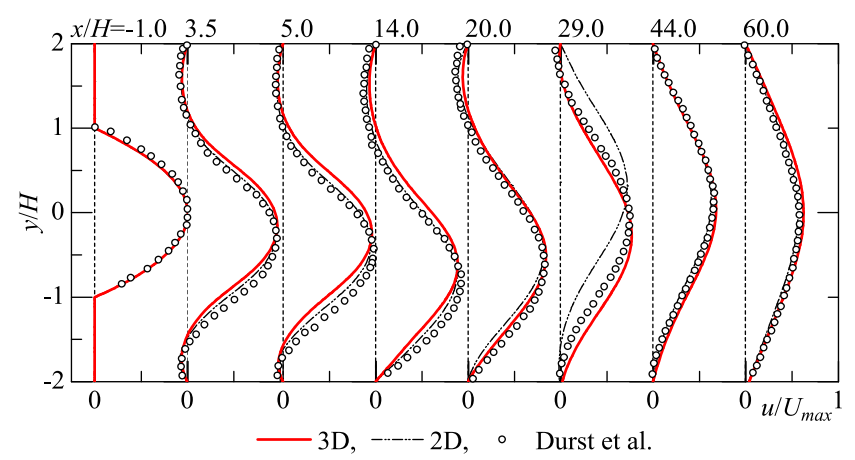

(b)

Figure 2. Comparison of streamwise velocity profile. (a) $E R=3, A R=24, \operatorname{Re}=186.7$; (b) $E R=2, A R=16, \operatorname{Re}=203.3$.

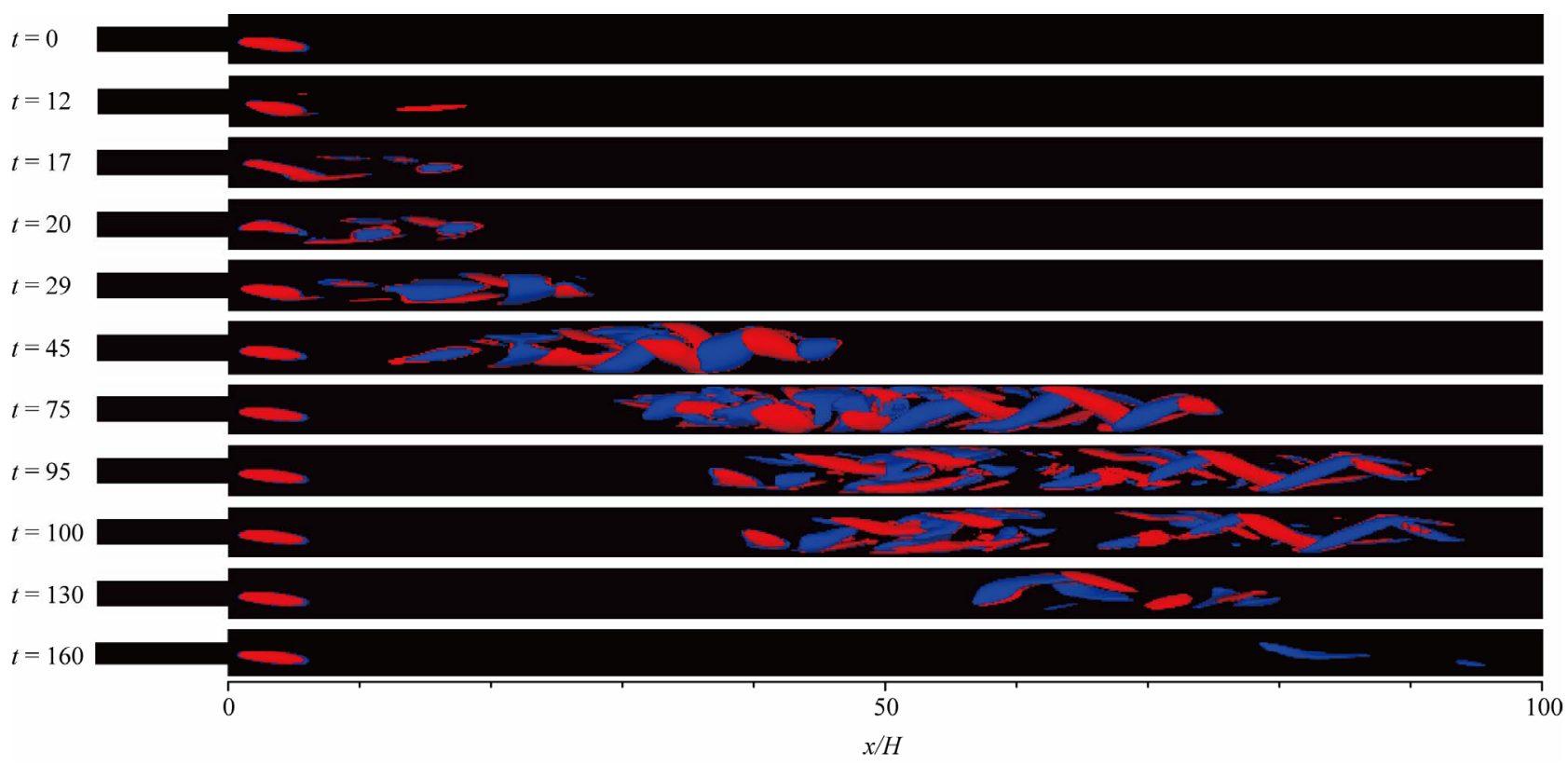

Figure 3. Side view of helicity isosurface $(E R=2, A R=8, \operatorname{Re}=200, \mathrm{St}=0.05, \square: h=-0.2, \square: h=+0.2)$. 


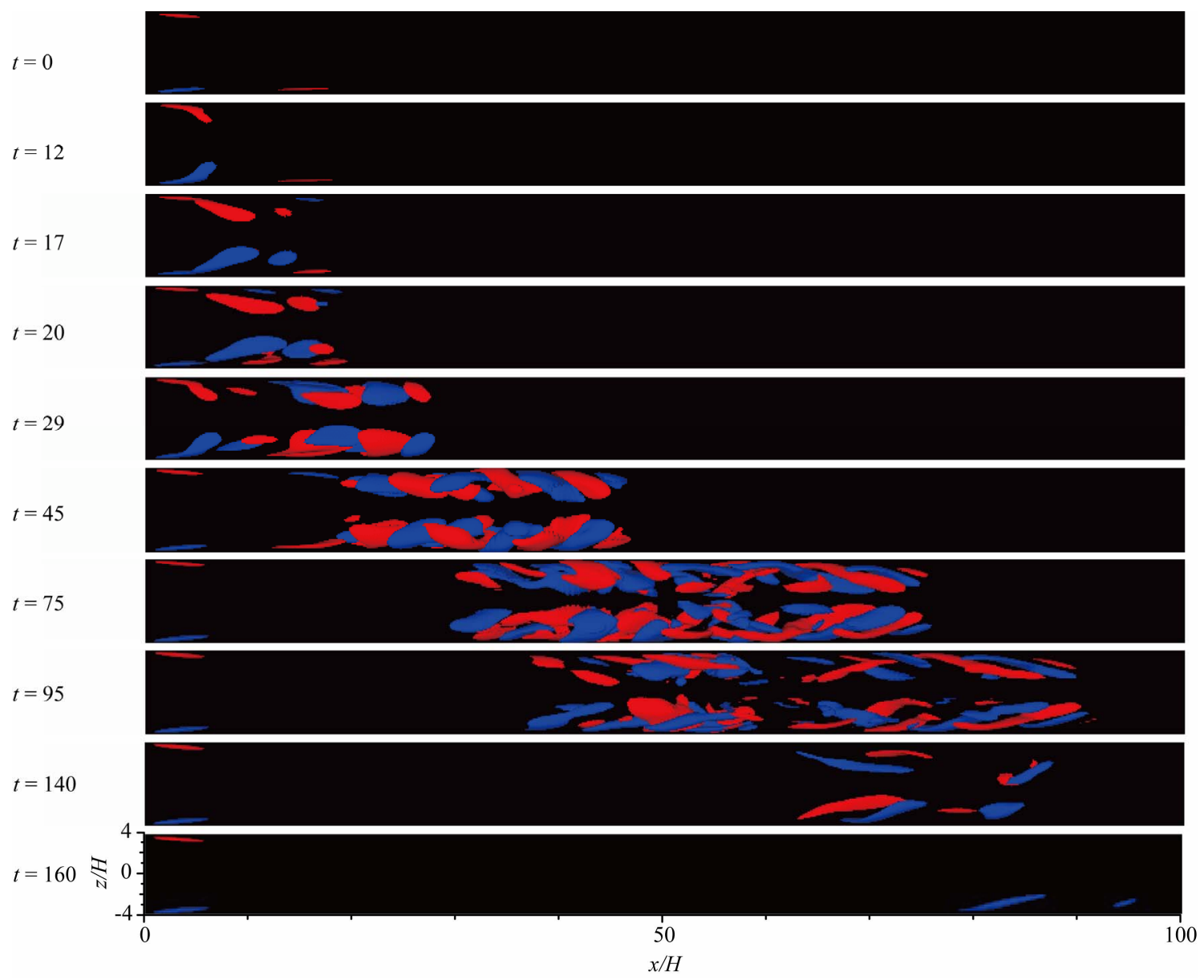

Figure 4. Bottom view of helicity isosurface $(E R=2, A R=8, \operatorname{Re}=200, \mathrm{St}=0.05, \square: h=-0.2, \square: h=+0.2)$

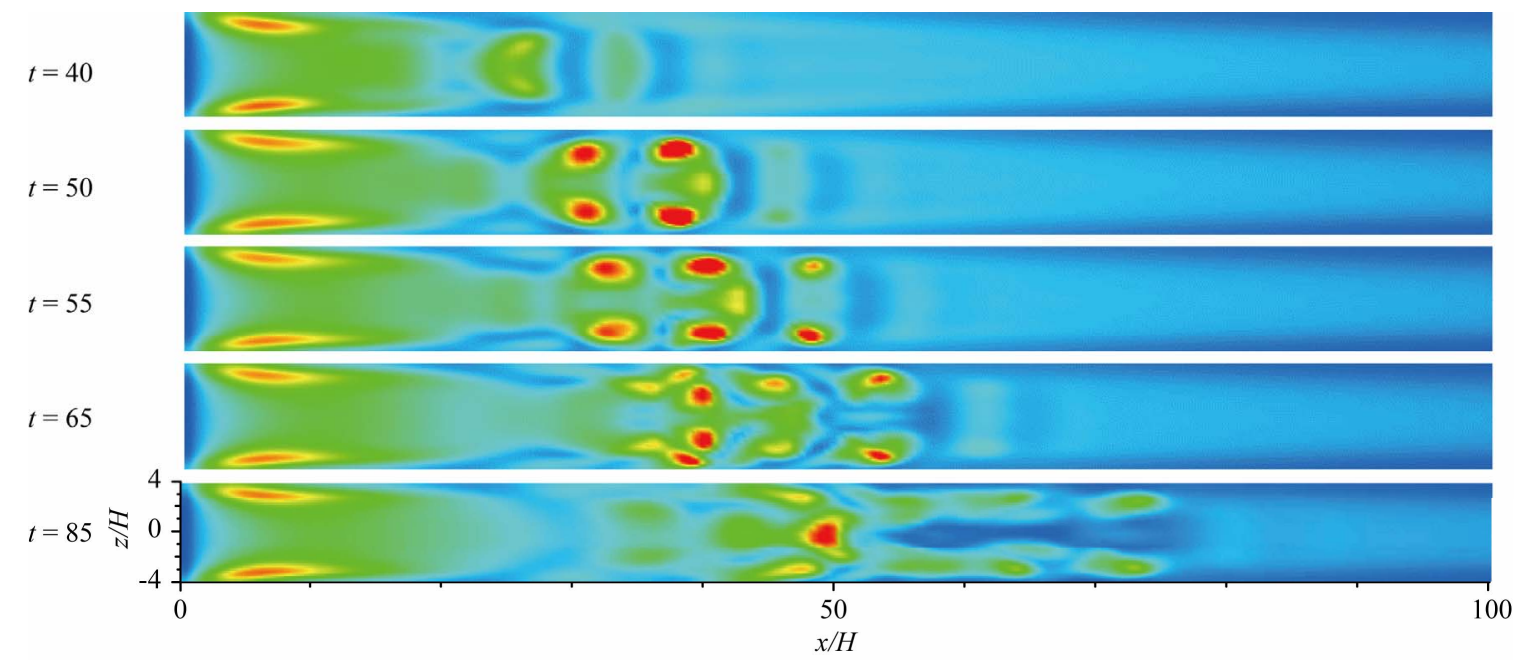

Figure 5. Local Nusselt number distribution on lower wall $(E R=2, A R=8, \operatorname{Re}=200, S t=0.05)$.

gradually decreases in accordance with the decay of the cloud vortices. On the other hand, effect of pulsation on the upper wall is observed from $t=40$ and slower than the lower wall. Nusselt number on the upper wall takes a peak near the side walls similar to that on the lower wall.
However the locations on the upper wall are slightly up-stream or downstream from them on the lower wall.

Time series of space averaged Nusselt number on the upper and lower walls is shown in Figure 7. It is clear that the transient response of heat transfer depends on 


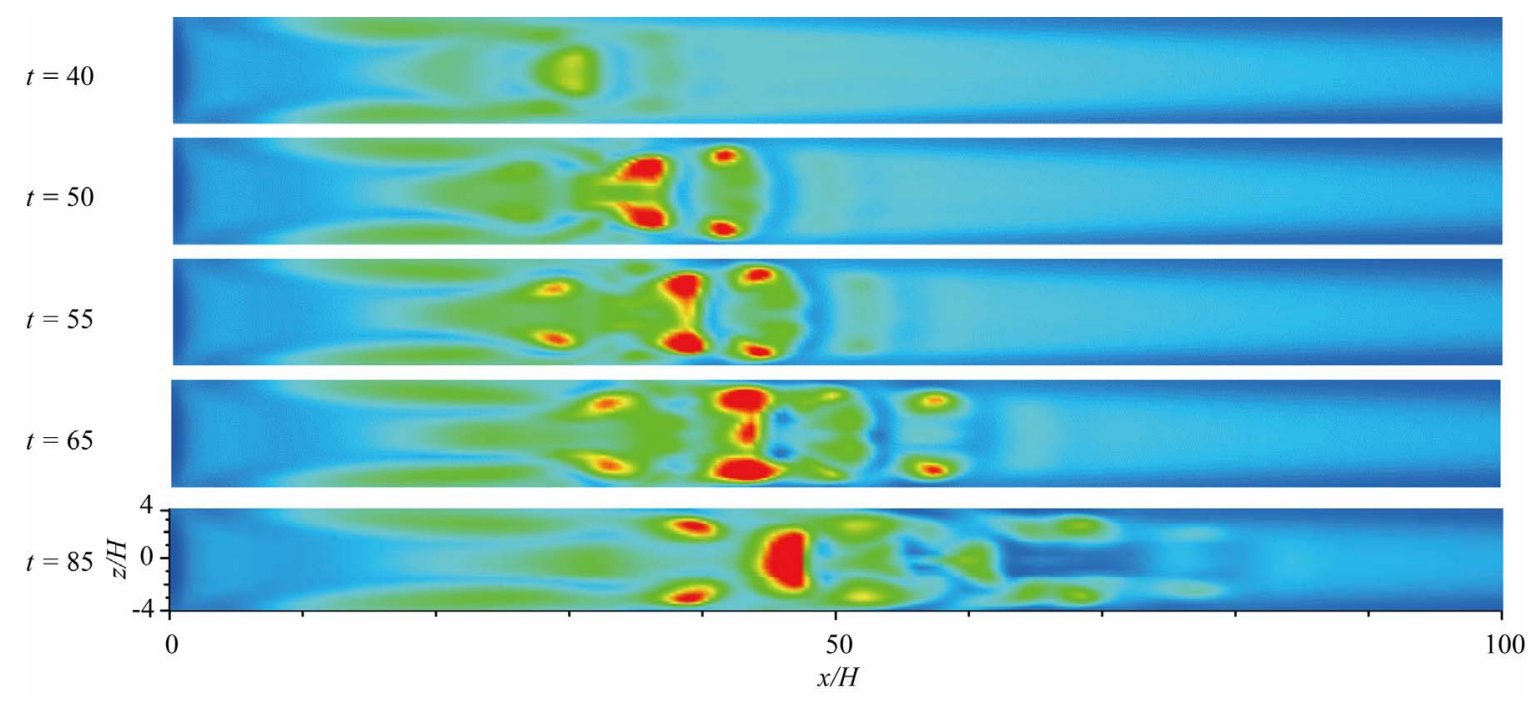

Figure 6. Local Nusselt number distribution on upper wall $(E R=2, A R=8, R e=200, S t=0.05)$.

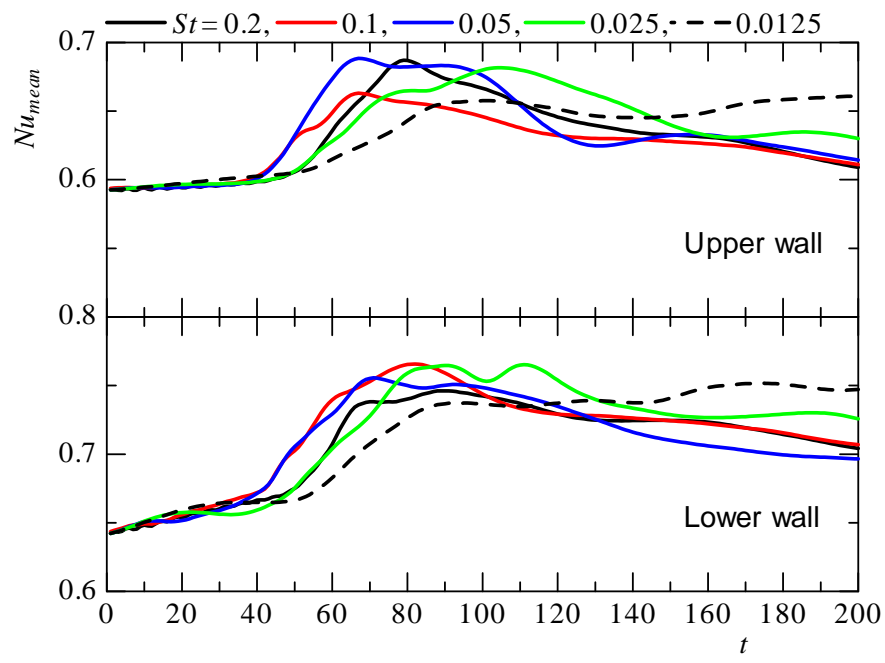

Figure 7. Time series of space averaged Nusselt number $(E R=2, A R=8, \operatorname{Re}=200, \mathrm{St}=0.05)$.

Strouhal number. Nusselt number of St $=0.05,0.10$ increases early and $\mathrm{Nu}_{\text {mean }}$ of $\mathrm{St}=0.025,0.20$ follows them. It seems that enhancement of heat transfer is almost independent on Strouhal number and the direction of wall.

\section{Concluding Remarks}

Numerical simulations of three-dimensional separated flow and heat transfer in the symmetric enlarged rectangular channel was conducted. Transient response of the flow and heat transfer in the channel with the fully developed inlet flow imposed one cycle of a pulsating fluctuation was investigated. Main results obtained are summarized as follows.

The vortices shed from behind of the step drift downstream along the side walls and sequentially induce some pairs of the streamwise vortices near the reattachment region. These cloud vortices incline the walls and gradu- ally decay.

Nusselt number on the walls takes a peak near the side walls on the upper and lower walls similarly. However the locations on the upper wall are slightly upstream or downstream from them on the lower wall.

In the case of St $=0.05,0.10$, space averaged Nusselt number increases early. However, enhancement of heat transfer is almost independent on Strouhal number of the pulsating fluctuation and the direction of wall.

\section{Acknowledgements}

These results were partly obtained with a supercomputer SX-9 of Cyberscience Center, Tohoku University.

\section{REFERENCES}

[1] D. E. Abbott and S. J. Kline, "Experimental Investiga- 
tions of Subsonic Turbulent Flow over Single and Double Backward Facing Steps," Journal of Basic Engineering, Vol. 84, No. 3, 1962, pp. 317-325.

[2] T. Ito, Y. Suematsu, Y. Simokawa and K. Tanaka, “A Study on the Bistable Fluid Amplifier Load Oscillator (1st Report, on the Flow Characteristics of the Fluid Amplifier)," Transactions of JSME, Vol. 39, No. 321, 1973, pp. 1599-1608. doi:10.1007/s11630-011-0437-X

[3] W. Cherdron, F. Durst and J. H. Whitelaw, “Asymmetric Flows and Instabilities in Symmetric Ducts with Sudden Expansions,” Journal of Fluid Mechanics, Vol. 84, No. 1, 1978, pp. 13-31. doi:10.1017/S0022112078000026

[4] I. J. Sobey, “Observation of Waves during Oscillatory Channel Flow,” Journal of Fluid Mechanics, Vol. 151, 1985, pp. 395-426. doi:10.1017/S0022112085001021

[5] A. Nishihara, K. Suzuki and Y. Inoue, "Numerical Analysis of Flow and Heat Transfer in an Enlarged Channel," Proceedings of 26th National Heat Transfer Symposium of Japan, Vol. 1, Sendai, 31 May-2 June 1989, pp. 37-39.

[6] M. Shapira, D. Degani and D. Weihs, "Stability and Existence of Multiple Solutions for Viscous Flow in Suddenly Enlarged Channels," Computers \& Fluids, Vol. 18, No. 3, 1990, pp. 239-258. doi:10.1016/0045-7930(90)90009-M

[7] R. M. Fearn, T. Mullin and K. A. Cliffe, "Nonlinear Flow Phenomena in a Symmetric Sudden Expansion,” Journal of Fluid Mechanics, Vol. 211, 1990, pp. 595-608. doi:10.1017/S0022112090001707

[8] T. Ota and Y. Toda, "Prediction of Laminar Heat Transfer and Flow in Plane Enlarged Channels (Heat Transfer 1990)," Hemisphere Publishing Corporation, New York, 1990, pp. 305-309.

[9] F. Durst, J. C. F. Pereira and C. Tropea, "The Plane Symmetric Sudden Expansion Flow at Low Reynolds Numbers,” Journal of Fluid Mechanics, Vol. 248, 1993, pp. 567-581. doi:10.1017/S0022112093000916

[10] T. Ota, H. Yanaoka and T. Hata, "Numerical Analysis of Laminar Flow and Heat Transfer in a Two-Dimensional Symmetrically Enlarged Channel," Transactions of JSME, Vol. 60B, No. 579, 1994, pp. 3930-3936. doi:10.1299/kikaib.60.3930

[11] S. Nakanishi, M. Sakurai and H. Osaka, "Numerical Study on Two-Dimensional Symmetric Sudden Expansion Channel Flow (Dynamic Characteristics)," Transactions of JSME, Vol. 61B, No. 589, 1995, pp. 3182-3189. doi:10.1299/kikaib.61.3182

[12] D. Drikakis, "Bifurcation Phenomena in Incompressible Sudden Expansion Flows,” Physics of Fluids, Vol. 9, No. 1, 1997, pp. 76-87. doi:10.1063/1.869174

[13] E. Schreck and M. Schäfer, "Numerical Study of Bifurcation in Three-Dimensional Sudden Channel Expansions," Computers \& Fluids, Vol. 29, No. 5, 2000, pp. 583-593. doi:10.1016/S0045-7930(99)00014-6

[14] M. Thiruvengadam, J. H. Nie and B. F. Armaly, "Bifurcated Three-Dimensional Forced Convection in Plane Symmetric Sudden Expansion," International Journal of Heat and Mass Transfer, Vol. 48, No. 15, 2005, pp. 31283139. doi:10.1016/j.ijheatmasstransfer.2005.02.019

[15] H. Yanaoka and T. Ota, “Three-Dimensional Numerical Simulation of Laminar Flow and Heat Transfer over Blunt Flat Plate in a Channel," Transactions of JSME, Vol. 62B, No. 596, 1996, pp. 1496-1501. doi:10.1299/kikaib.62.1496

[16] H. Yanaoka and T. Ota, “Three-Dimensional Numerical Simulation of Unsteady Flow and Heat Transfer over Blunt Flat Plate in Channel," Transactions of JSME, Vol. 62B, No. 599, 1996, pp. 2745-2750. doi:10.1299/kikaib.62.2745

[17] T. Ota, H. Yanaoka, K. Shibuya, M. Nakajima and H. Yoshikawa, "Numerical Simulation of Separated Flow and Heat Transfer in a Rectangular Channel with Sudden Expansion," Proceedings of ASME FEDSM, Boston, 11-15 June 2000, Paper No. FEDSM00-11004.

[18] H. Iwai, K. Nakabe and K. Suzuki, "Flow and Heat Transfer Characteristics of Backward-Facing Step Laminar Flow in a Rectangular Duct," International Journal of Heat and Mass Transfer, Vol. 43, No. 3, 2000, pp. 457471. doi:10.1016/S0017-9310(99)00140-4

[19] H. Yoshikawa, T. Kai, M. Munekata and H. Ohba, "Effects of Pulsation on Separated Flow and Heat Transfer in Enlarged Channel," Journal of Thermal Science, Vol. 20, No. 1, 2011, pp. 70-75. doi:10.1007/s11630-011-0437-x

[20] C. Fan and B. Chao, "Unsteady, Laminar, Incompressible Flow through Rectangular Ducts,” Zeitschrift für angewandte Mathematik und Physik (ZAMP), Vol. 16, No. 3, 1965, pp. 351-360. doi:10.1007/BF01591915

[21] A. A. Amsden and F. H. Harlow, "A Simplified MAC Technique for Incompressible Fluid Flow Calculations," Journal of Computational Physics, Vol. 6, No. 2, 1970, pp. 322-325. doi:10.1016/0021-9991(70)90029-X 


\section{Nomenclature}

$A R \quad$ Aspect ratio $\left(=W_{3} / H\right)$

ER Expansion ratio $\left(=W_{2} / W_{1}\right)$

h Helicity

$H \quad$ Step height

$\mathrm{Nu} \quad$ Nusselt number $(=\alpha H / \lambda)$

$\mathrm{Nu}_{\text {mean }} \quad$ Space averaged Nusselt number

$p \quad$ Pressure

Pr Prandtl number $(=v / \alpha)$

$q_{\mathrm{w}} \quad$ Heat flux

Re Reynolds number $(=U H / v)$

St Strouhal number $(=f H / U)$

$\begin{array}{ll}t & \text { Time } \\ \boldsymbol{u} & \text { Velocity vector }(u, v, w) \\ U & \text { Mean velocity at inlet } \\ U_{\max } & \text { Maximum velocity at inlet } \\ \boldsymbol{x} & \text { Coordinates }(x, y, z)\end{array}$

\section{Greek Letters}

$\alpha \quad$ Heat transfer coefficient

$\theta \quad$ Temperature

$\lambda$ Heat conductivity

$v \quad$ Kinematic viscosity 